TRANSACTIONS OF THE

AMERICAN MATHEMATICAL SOCIETY

Volume 211,1975

\title{
STRONGLY PRIME RINGS
}

BY

\section{DAVID HANDELMAN AND JOHN LAWRENCE}

ABSTRACT. A ring $R$ is (right) strongly prime (SP) if every nonzero twosided ideal contains a finite set whose right annihilator is zero. Examples are domains, prime Goldie rings and simple rings; however, this notion is asymmetric and a right but not left $\mathrm{SP}$ ring is exhibited. All SP rings are prime, and every prime ring may be embedded in an SP ring. SP rings are nonsingular, and a regular SP ring is simple; since faithful rings of quotients of $\mathrm{SP}$ rings are $\mathrm{SP}$, the complete ring of quotients of an SP ring is simple.

All SP rings are coefficient rings for some primitive group ring (a generalization of a result proved for domains by Formanek), and this was the initial motivation for the ir study. If the group ring $R G$ is SP, then $R$ is $\mathrm{SP}$ and $G$ contains no nontrivial locally finite normal subgroups.

Coincidentally, SP rings coincide with the ATF rings of Rubin, and so every SP ring has a unique maximal proper torsion theory, and $(0)$ and $R$ are the only torsion ideals. ( 1 )

A list of questions is appended.

Introduction. In [3] Formanek showed that if $D$ is a domain and $G$ is a suitable free product of groups, then the group ring $D G$ is primitive. As a generalization of Formanek's result, the second author [12] showed that the domain could be replaced by a prime ring with a type of finiteness condition: a 'strongly prime' ring.

Although primitive group rings were the original motivation for strongly prime (SP) rings, it became apparent that these rings are interesting by themselves. §II deals with elementary properties of SP rings: every prime ring may be embedded in an SP ring; all SP rings are nonsingular and only the artinian SP rings have a minimal right ideal. We show that SP plus regular implies simple, a special case of Kaplansky's (still open) conjecture that a prime regular ring is primitive.

The third section discusses a possible characterization for SP group rings, and proves the above generalization of Formanek's result.

Little is known about the structure of prime regular self-injective rings. 1973.

Presented to the Society, October 27, 1973; received by the editors October 1,

AMS (MOS) subject classifications (1970). Primary 16A12; Secondary 16A08, 16A12, 16A48.

(1) At the time the authors submitted their abstract to the Society, they were unaware of Rubin's results. 
The maximal ring of quotients of an SP ring is simple (and SP), and we may assign a certain finite or countable cardinal to SP rings; thereby we may classify simple self-injective rings.

The final section considers torsion and near torsion theories on SP rings -there is a unique maximal proper torsion theory on SP rings. It is shown that the so-called ATF rings of [14] are exactly the SP rings; a surprising result considering the differences in motivation.

The authors would like to thank Professors P. M. Cohn, I. Connell and J. Lambek, as well as Michael Josephy and A. Rahman for their assistance and encouragement.

Near the completion of this work, the authors were chagrined to note that some of the results had been obtained (independently) in the $\mathrm{Ph}$. $\mathrm{D}$. thesis of Viola-Prioli.

I. Definitions and examples. All rings are associative, with identity, and usually denoted $R$. If $A$ is a subset of the ring $R$ of a right $R$-module, then we define the (right) annihilator of $A(A n n(A))$ to be the right ideal

$$
\operatorname{Ann}(A)=\{r \in R \mid A r=(0)\} \text {. }
$$

We adopt the symbol $R^{\circ}$ to denote the nonzero elements of $R$.

If $A$ is a right ideal in $R$, and $r$ is an element of $R$, we define $r^{-1} A$ to be the right ideal

$$
r^{-1} A=\{s \in R \mid r s \in A\} .
$$

In this notation, Ann $(\{r\})$ becomes $r^{-1}(0)$, which we abbreviate to $r^{-1} 0$.

Recall that a ring $R$ is prime if, for given $r, t \in R$; there exists $s \in R$ such that $r s t \neq 0$. If for each nonzero element $r$ we can restrict the choice of the $s$ to a finite set (independent of $t$, but depending on $r$ ), then we have a ring that is stronger than prime. Define a (right) insulator for $r \in R^{\circ}$ to be a finite subset of $R, S(r)$, such that

$$
\text { Ann }(\{r s \mid s \in S(r)\})=(0) \text {. }
$$

$R$ is (right) strongly prime (SP) if each nonzero element of $R$ has a right insulator. That is, for each $r \in R$; there is a finite set $S(r)$ such that for $t \in R$,

$$
\{r s t \mid s \in S(r)\}=\{0\} \text { implies } t=0 \text {. }
$$

Although prime is a symmetric notion, strongly prime is not (an example of a left but not right SP ring will be given). However, we will generally work on the right, and 'strongly prime' and 'insulator' will denote right strongly prime and right insulator, respectively. 
Alternate definition of strongly prime. $R$ is strongly prime if every nonzero two-sided ideal of $R$ contains a finitely generated left ideal whose right annihilator is zero.

The equivalence of the two definitions is straightforward.

It follows immediately from the alternate definition that simple rings, and prime left noetherian rings are right SP. They are also left SP.

A domain is SP: choose a nonzero element $d$; then $\{d\}$ is an insulator for every nonzero element. When the same insulator can be chosen for every element of $R$, we call it a uniform insulator, and the ring is uniformly $S P$. If for a fixed integer $n$, we can assign to each nonzero element of $R$ an insulator (not necessarily a uniform insulator) with $n$ or less elements, then $R$ is bounded $S P$, of bound $n$ or less.

To obtain more examples of SP rings, we utilize the following results. Recall that the DCC on right annihilators allows us to choose a minimal element from every nonvoid collection of right annihilator ideals, and is equivalent to ACC on left annihilators.

Proposition I.l. If $R$ is a prime ring with DCC on right annihilators, then $R$ is (right) strongly prime.

Proof. Choose $r \in R$ : From the collection of right annihilator ideals of the form Ann ( $\{r \mid s \in I\})$ where $I$ runs over all finite subsets of $R$ containing the identity, choose a minimal element $M$. If $M \neq(0)$, we can find an $m \epsilon$ $M$ and $q \in R$ such that rqm $\neq 0$, since $R$ is prime, and we construct a smaller element than $M$, contradiction. Hence $M=(0)$, so $r$ has an insulator.

Proposition 1.2. If $R$ is an $n \times n$ matrix ring over an Ore domain, then $R$ is bounded strongly prime of bound $n$. Also, $R$ has uniform bound $n^{2}$.

Proof. For any nonzero element of $R$, a set of matrix units in some row is an insulator. Hence the bound is no greater than $n$. As we will see, the bound is not less than $n$. Finally, since the set of matrix units is an insulator, $R$ has uniform bound $n^{2}$.

From Proposition 1.2, we see that if $D$ is a division ring, then $M_{n}(D)$ is bounded SP with bound exactly $n . M_{n}(D)$ is also uniformly SP of bound $n^{2}$ (the matrix units form a uniform insulator).

From [7] a prime left Goldie ring has ACC on both right and left annihilator ideals, and thus by Proposition I.1 is right and left SP. More can be shown-as a consequence of the Faith-Utumi theorem [10], prime left Goldie rings are uniformly SP and hence bounded SP. In view of this, we obtain 
Proposition I.3. If $R$ is a prime right Goldie ring, then $R$ is a domain if and only if $R$ has bound equal to one.

We now consider some pathological properties that SP rings may have.

Example 1. We construct a ring that is right strongly prime but not left strongly prime.

Let $Z_{2}$ denote the field of two elements, and let $D=Z_{2}\left[x_{i}\right]$ (where $i$ runs over the positive integers) be the free (noncommuting) $Z_{2}$-algebra on $\left\{x_{i}\right\}$. Let $l$ be the two-sided ideal in $D$ generated by monomials of the form $x_{i} x_{j} x_{k}$ with $i>j>k$. Set $R=D / l$.

We show $R$ is right SP. If $m$ is a nonzero monomial in $R$ of the form $x_{j_{1}} x_{j_{2}} \cdots x_{j_{k}}$ (we have, of course identified the $x_{i}$ with their images in $R$ ), $S(m)=\left\{x_{j_{k}} x_{1}\right\}$ is an insulator for $m$ in $R$. (It suffices to observe that since $I$ is generated by monomials, if, for $m_{i}$ monomials in $D, \Sigma m_{i}$ belongs to $I$, then each $m_{i}$ belongs to $I_{\text {.) }}$ If $r=m_{1}+m_{2}+\cdots+m_{n}$ is a sum of nonzero monomials in $R$, choose $m_{1}$ to be a monomial of maximal degree among the $m_{i}$; then $S\left(m_{1}\right)$ is an insulator for $r$.

However, $x_{1}$ has no left insulator: for a finite subset $\left\{r_{j}\right\}$ of $R$, we can find $n$ sufficiently large so that $x_{n+1} x_{n} r_{j} x_{1}=0$ for all $j$. Thus $R$ is bounded right SP, but not left SP.

Example 2. We find a chain of SP rings $R_{n}$ such that $U R_{n}$ is not SP. (Thus the class of SP rings is not inductive.)

Let $S_{n}=\left\{x_{1}, x_{2}, \ldots, x_{2 n}\right\}$, and set $D_{n}=Z_{2}\left[S_{n}\right]$, the free $Z_{2}$-algebra on $2 n$ variables. Define a sequence of ideals $\left\{I_{n}\right\}$ in $D_{n}$ inductively by

$$
\begin{aligned}
& I_{1}=(0), \\
& I_{n+1} \text { is the ideal in } D_{n+1} \text { generated by } \\
& \qquad I_{n} \cup\left\{x_{i} x_{j} \mid i \text { or } j=2 n-1 \text { and } i, j<2 n\right\} .
\end{aligned}
$$

Note that $I_{n}=D_{n} \cap I_{n+1}$.

Set $R_{n}=D_{n} / I_{n}$. It is easily seen that $\left\{x_{2 n}\right\}$ is a uniform insulator in $R_{n}$. Also, $R_{n}$ is contained in $R_{n+1}$ (via the canonical map). However $R=$ $\bigcup_{R_{n}}$ is not SP since there is no finite set $S$ such that

$$
\left\{x_{1} s x_{n} \mid s \in S\right\} \neq\{0\} \text { for all } n,
$$

so $x_{1}$ has no right insulator in $R$.

Example 3. We construct an SP ring that is not bounded.

Proposition I. 4. If $R=M_{n}(F)$ where $F$ is a field, and $e_{i j}$ the matrix units, let $r=e_{11}+e_{22}+\cdots+e_{m m}$ with $m<n$. Suppose $\left\{s_{i}\right\}_{i=1}^{k^{i j}}$ is an insulator for $r$ in $R$. Then $k \geq n / m$. 
Proof. Each rs ${ }_{i}$ must be of the form

$$
\left[\frac{R_{i(m \times n)}}{0(n-m \times n)}\right] \text {. }
$$

For each $i$ we have $m$ equations (each row in $R_{i}$ ) and we thus have a total $k m$ equations. If $n>k m$, then we can find nonzero $t$ in $R$ such that $r s_{i} t=$ 0 for all $i$; hence $k m \geq n$.

Let $R$ be the direct limit of matrix rings over a field via the embeddings $M_{n}(F) \rightarrow M_{2 n}(F)$ given by

$$
\left[\begin{array}{ll}
a & b \\
c & d
\end{array}\right] \rightarrow\left[\begin{array}{llll}
a & 0 & b & 0 \\
0 & a & 0 & b \\
c & 0 & d & 0 \\
0 & c & 0 & d
\end{array}\right]
$$

Since $R$ is a direct limit of simple rings, $R$ is simple, hence SP. Any insulator of $e_{11}$ in $M_{n}(F)$ has cardinality at least $n$, and by the preceding proposition, this does not decrease on taking the image of $e_{11}$ (in $M_{n}(F)$ ) in the direct limit. Thus, the cardinalities of the insulators of limages in $R$ of $\left.e_{11} \in M_{n}(F)\right\}$ are not bounded.

II. Elementary properties.

Proposition II.1. Strongly primeness is a Morita invariant.

Proof. Let $R$ be SP and $e$ a nonzero idempotent in $R$. If $\left\{f_{i}\right\}$ is an insulator in $R$ for some nonzero element of the form ere, then $\left\{e f_{i} e\right\}$ is an insulator for ere in $e$ Re. Thus $e$ Re is SP.

To show $M_{n}(R)$ is SP if $R$ is, choose a nonzero matrix $B$ in $M_{n}(R)$, and say its $p q$ component, $b_{p q}$, is nonzero. Let $\left\{t_{k}\right\}$ be an insulator for $b_{p q}$ in $R$. Then if $A$ is a nonzero matrix, with nonzero $a_{i j}$ in the $i j$ component, we have that the $p j$ entry of $B\left(e_{q i} t_{k}\right) A$ is $b_{p q} t_{k} a_{i j}$. Hence $\left\{e_{i j} t_{k}\right\}_{i, j, k}$ is an insulator for $B$.

The singular ideal of $R$ (denoted $Z(R)$ ) is the ideal composed of elements whose right annihilator is an essential right ideal.

Proposition II.2. The singular ideal of a strongly prime ring is zero.

Proof. Suppose $R$ is SP, and $r \in Z(R) \cap R$ : Let $\left\{r_{i}\right\}$ be an insulator for $r$. Since $Z(R)$ is an ideal, there exist essential right ideals $E_{i}$ such that $r_{i} E_{i}=(0)$. Thus

$$
\pi_{i}\left(\bigcap_{j} E_{j}\right)=(0) \text { for all } i
$$


The intersection of finitely many essential right ideals is nonzero; this contradicts the insulator property of the $\left\{r_{i}\right\}$. So, $Z(R)=(0)$.

In [9] the question is asked: Is a prime regular ring primitive? It happens that regular SP rings are not just primitive but simple (no two-sided ideals).

Proposition II.3. A regular strongly prime ring is simple.

Proof. In a regular ring $R$ every finitely generated left ideal is of the form $R e$, where $e$ is an idempotent. $R e$ has $(0)$ as its right annihilator only if $e=1$. From the alternate definition of SP, it is clear that every two-sided nonzero ideal contains 1 ; thus $R$ is simple.

If every proper finitely generated left ideal has nonzero right annihilator, then we call the ring an $F$-ring. All regular rings are $F$-rings, and Proposition II. 3 is a special case of the result that an SP F-ring is simple.

Corollary. A regular ring is simple if and only if it is strongly prime.

If $R$ is regular and uniformly SP of bound 1 , then $R$ is a division ring. (Proof. If $\{x\}$ is the uniform insulator, then $x^{-1} \in R^{\circ}$; if $a, b \in R^{\circ}$, then $x^{-1} b \in R$, so $a b=a x x^{-1} b \neq 0$; thus $R$ is a regular domain.) If $R$ is regular, and uniformly SP of bound $n$, it seems likely that $R$ is a matrix ring over a division ring and $n$ is a square.

We now show that any prime ring (indeed, any ring that is torsion-free as a module over its centre) may be embedded in a strongly prime ring.

Let $R$ be a prime ring with centre $D$; since $R$ is torsion-free as a module over its centre, the nonzero elements of $D$ may be inverted to yield a prime ring whose centre is a field.

Proposition II.4 ('Embedding Theorem'). Every prime ring may be embedded in a strongly prime ring.

Proof. By the preceding comment, we may assume the centre of the prime ring $R$ is a field $F$. Let $F[x]$ denote the polynomial ring in one variable over $F$. If $\left\{1, r_{k}\right\}$ is a basis for $R$ over $F$, a well-known result asserts that

$$
\left\{r_{k_{1}} x^{i 1} \cdots r_{k_{n}} x^{i} n \mid r_{k_{j}} \neq 1 \text { for } j>1, i_{j} \neq 0 \text { for } j \leq n-1\right\}
$$

is a basis for the free product of $F$-algebras, $T=R{ }_{F} F[x]$. Thus $R$ may be imbedded in $T$. It is easily seen that $\{x\}$ is a uniform insulator for $T$.

By a similar argument, the free product of any two $F$-algebras with 1 is SP; further by taking a suitable subring it becomes clear that any prime ring can be embedded in a strongly prime ring with the same centre. 
III. Group rings. In this section we obtain some elementary results on SP group rings, and conjecture a characterization of them. We also show that every SP ring is the coefficient ring of some primitive group ring.

The letter $G$ denotes a group throughout this section. Recall that if every finitely generated subgroup of $G$ is finite, then $G$ is said to be locally finite.

Proposition III.1. (a) If the group ring $R G$ is strongly prime then $R$ is strongly prime and $G$ contains no locally finite normal subgroups other than $\{1\}$;

(b) If $G$ is torsion-free abelian and $R$ is strongly prime then $R G$ is strongly prime.

Proof. (a) To show $R$ is SP, choose $r \in R^{\circ}$, and let $\left\{s_{i}\right\}$ be an insulator for $r$ in $R G$. We may write $s_{i}=\Sigma_{j} r_{i j} g_{i j}$ where $r_{i j} \in R$ and $g_{i j} \in G$. Then $\left\{r_{i j}\right\}_{i, j}$ is an insulator for $r$ in $R$.

If $G$ contains a nontrivial locally finite normal subgroup $N$, choose $g \epsilon$ $N, g \neq 1$. We show $g-1$ has no insulator in $R G$. If $\left\{s_{i}\right\}$ is an isulator for $g-1$, then $\left\{g_{k}\right\}=\bigcup_{i} \operatorname{support}\left(s_{i}\right)$ is also an insulator for $g-1$. Define $H$ to be the group generated by $\left\{g_{k}^{-1} g g_{k}\right\}_{k} . H$ is a subgroup of $N$, and is finitely generated, hence finite. Therefore, by [10, Lemma 2 (c), p. 154], there exists nonzero $s$ in $R G$ such that $\left(g_{k}^{-1} g g_{k}-1\right) s=0$ for all $k$. But $g_{k}\left(g_{k}^{-1} g g_{k}-1\right)=(g-1) g_{k}$; so $(g-1) g_{k} s=0$ for all $k$, contradicting the insulator property of $\left\{g_{k}\right\}$. Thus if $R G$ is SP, $G$ contains no nontrivial locally finite normal subgroups.

(b) Since $G$ is torsion-free abelian, it can be ordered. Suppose $s \in R G$, with $s=\sum_{i=1}^{k} r_{i} g_{i}$, where $r_{i} \in R$; and $g_{k}$ is maximal among $\left\{g_{i}\right\}$. Then any insulator for $r_{k}$ in $R$ is an insulator for $s$ in $R G$.

The first part of (a) and (b) are, in effect, obtained in [15]. The author of [15] asks (in effect) if $R$ is SP and $G$ contains no finite normal subgroup, then is RG SP? No; let $F$ be a field of characteristic zero and $S$ the permutation group on the integers (each element of $S$ changes the positions of only finitely many members), then $S$ is itself locally finite, so FS is not SP, but $F S$ is prime (in fact primitive).

Connell [1] has shown that the group ring $R G$ is prime if and only if $R$ is prime and $G$ contains no (nontrivial) finite normal subgroups. Part (a) suggests the following generalization of Connell's result: The group ring $R G$ is strongly prime if and only if $R$ is strongly prime and $G$ contains no locally finite normal subgroups (other than 1). Unfortunately, efforts to prove the converse of (a) seem to run into difficulties paralleling those of the zero-divisor problem. 
We now consider a relation between primitive group rings and SP rings.

In [3] Formanek showed that if $R$ is a domain and $G=A * B$ is a free product of nontrivial groups $A$ and $B$ not both of order 2 , and $\#(G) \geq \#(R)$, then $R G$ is primitive. We shall generalize this from domains to SP rings. The proof is similar to that in [3].

A right ideal of $R, M$, is said to be comaximal if $M$ is proper and $M+J$ $=R$ for any two-sided ideal $J$ that is nonzero.

Proposition III.2. A ring is (right) primitive if and only if it contains a comaximal right ideal.

For a group $G$, set $G^{\prime}=G-\{1\}$. Let $G=A * B$; we say that an element $g$ of $G$ is of type $A A$ and has length $2 n+1$ if it has the reduced form

$$
g=a_{1} b_{1} a_{2} b_{2} \cdots a_{n} b_{n} a_{n+1} \text { where } a_{i} \in A^{\prime} \text { and } b_{i} \in B^{\prime} \text {. }
$$

We define elements of types $A B, B A$, and $B B$, and their lengths in a similar manner.

Proposition III.3. If $R$ is strongly prime and $G=A * B$ is a free product of nontrivial groups $A$ and $B$ (not both of order 2 ), and $\#(G) \geq \#(R)$, then the group ring $R G$ is primitive and strongly prime.

Proof. We first show that $R G$ is SP. Choose nonzero $s$ in $R G$. Let $r$ be the coefficient of a group element of maximal length in the. support of $s$, and let $\left\{r_{i}\right\}$ be an insulator for $r$ in $R$. Set $S=\left\{r_{i} a a, r_{i} a b, r_{i} b a, r_{i} b b\right\}$ where $a$ and $b$ are fixed elements of $A^{\prime}$ and $B^{\prime}$ respectively. Then $S$ is an insulator for $s$ in $R G$.

We now consider the primitivity. We may assume that $\#(A) \geq \#(B)>1$, and $\#(A)>2$.

Case 1. $A$ is infinite.

Here we have $\#(R G)=\#(G)=\#(A)=\#\left(A^{\prime}\right)$. Let $N$ denote the positive integers.

Let $W: A^{\prime} \rightarrow(R G-\{0\}) \times N$ be a bijection of sets and let $A^{*}=$ $W^{-1}((R G-\{0\}) \times 1)$. For each $a \in A^{*}$, there corresponds a unique element in $R G-\{0\}$, which we denote by $r(a, 1)$. Let $a(n)$ represent the element mapped onto $r(a, 1) \times n$. For $a \in A^{*}$, let $g(a)$ be an element of maximal length in the support of $r(a, 1)$, and suppose that in $r(a, 1), g(a)$ has coefficient $r$. Let $b$ be a fixed element of $B^{\prime}$ and set $h(a)$ equal to

$$
\begin{aligned}
& \sum\left(b a(i) b r(a, 1) r_{i}+a(i) b r(a, 1) a(i) r_{i}\right)+1 \\
& \quad \text { if } g(a) \text { is of type } A B \text { or } g(a)=1 \text {, and where the sum } \\
& \text { is taken through }\left\{r_{i}\right\} \text {, the insulator for } r \text { in } R \text {. }
\end{aligned}
$$


Similarly, we define $h(a)$ equal to

$$
\begin{array}{ll}
\sum\left(a(i) b r(a, 1) b r_{i}+b a(i) b r(a, 1) r_{i}\right)+1 & \text { if } g(a) \text { is of type } A A ; \\
\sum\left(a(i) r(a, 1) b r_{i}+b a(i) r(a, 1) r_{i}\right)+1 & \text { if } g(a) \text { is of type } B A \\
\sum\left(b a(i) r(a, 1) r_{i}+a(i) r(a, 1) a(i) r_{i}\right)+1 & \text { if } g(a) \text { is of type } B B .
\end{array}
$$

We show that the right ideal generated by all the $h(a)$ is comaximal in $R G$.

If $x \in R G$, then an element of maximal length in the support of $h(a) x$ begins with either $b a(i)$ or $a(i)$ in its reduced form. Hence an equation of the form $\Sigma h\left(a_{i}\right) x_{i}=1$ is impossible, since group elements of maximal length in the support of $h\left(a_{i}\right) x_{i}$ begin with either $b a_{i}(n)$ or $a_{i}(n)$, for some $n$.

Let $M$ be the right ideal of $R G$ generated by all the $h(a)$. The above result shows that $M$ is proper, and the other condition for comaximality is clear from the definition of the $h(a)$.

Case 2. $A$ is finite.

In this case, we have a bijection $W: N \rightarrow(R G-\{0\}) \times N$. Let $N^{*}=$ $W^{-1}((R G-\{0\}) \times 1)$. To each $n \in N^{*}$, there corresponds a unique element in $R G-\{0\}$, which we denote by $r(n)$. Let $n(k)$ denote the element of $N$ mapped onto $r(n) \times k$.

For $n=n(1) \in N^{*}$, let $g(n)$ be an element of maximal length in the support of $r(n)$, and suppose that in $r(n), g(n)$ has coefficient $r$.

Let $a$ and $c$ be fixed elements of $A^{\prime}$, and $b$ a fixed element of $B^{\prime}$. Given $n=n(1) \in N^{*}$, set $h(n)$ equal to

$$
\begin{gathered}
\sum\left((a b)^{n(i)} c b r(n) a r_{i}+b(a b)^{n(i)} c b r(n) r_{i}\right)+1 \\
\text { if } g(n) \text { is of type } A B \text { or } g(n)=1 \text {, and } \\
\text { as before, }\left\{r_{i}\right\} \text { is an insulator for } r .
\end{gathered}
$$

Similarly, we define

$$
\begin{array}{ll}
h(n)=\sum\left(b(a b)^{n(i)} c b r(n) r_{i}+(a b)^{n(i)} c b r(n) b r_{i}\right)+1 & \text { if } g(a) \text { is of type } A A ; \\
h(n)=\sum\left((a b)^{n(i)} c r(n) a r_{i}+b(a b)^{n(i)} c r(n) r_{i}\right)+1 & \text { if } g(a) \text { is of type } B B ; \\
h(n)=\sum\left(b(a b)^{n(i)} c r(n) r_{i}+(a b)^{n(i)} c r(n) b r_{i}\right)+1 & \text { if } g(a) \text { is of type } B A .
\end{array}
$$

Let $M$ be the right ideal generated by all the $h(n)$. As in Case $1, M$ is comaximal, so $R G$ is primitive.

This complete the proof of the proposition.

Remark 1. In [12], an example of a prime semiprimitive ring $R$ is given 
with the property that if $G$ is any group, then $R G$ is not primitive.

Remark 2. Let $R$ be a prime ring, and suppose its centre is a field $F$. If $R$ contains a nonzero two-sided nil ideal that is locally nilpotent as an $F$-algebra, then for any group $G$, the group ring $R G$ is not semiprimitive, and hence not primitive [12]. Thus a ring with such a nil ideal is not strongly prime. However not all nil algebras are locally nilpotent (a consequence of the Golod-Shafarevitch theorem) so the question remains: Can a strongly prime ring contain a nonzero nil ideal?

IV. Rings of quotients. We use the definition of (right) ring of quotients (or quotient ring) given on p. 99 of [10]. For $R$ and $S$ rings, if $R \subset S$ then $S$ is a (right) essential extension ring of $R$ if $R$ is an essential right $R$-submodule of $S$. With these definitions, every ring of quotients is an essential extension ring. For a ring $R$, we denote its complete ring of quotients [10, p. 94] by $Q$. If $Z(R)=(0)$, then $Q$ is the largest essential extension ring of $R$.

It is easy to show that if $R$ is prime, then any essential extension ring of $R$ is prime. Analogously, we have

Proposition IV.l. If $R$ is (right) strongly prime, then any essential extension ring of $R, S$, is strongly prime. Further, if $R$ is bounded strongly prime of bound $n$, then $S$ is bounded of bound $n$ or less.

Proof. Choose $s \in S$ : There exists $r \in R$ such that $s r \in R$; hence there exists a finite set $\left\{r_{i}\right\} \subset R$ such that $\operatorname{Ann}\left(\left\{s r r_{i}\right\}_{i}\right)=(0)$. For $t \in S^{\circ}$, there exists $r^{\prime} \in R$ such that $t r^{\prime} \in R^{\prime}$; so we may find an $i_{0}$ such that $s r r_{i} t r^{\prime} \neq 0$. Therefore, $s r r_{i} t \neq 0$, so $\left\{r_{i}\right\}$ is an insulator for $s r$ in $S$, and $\left\{r r_{i}\right\}$ is an insulator for $s$.

Corollary 1. The complete ring of quotients of a strongly prime ring is simple.

Proof. We know from Proposition II.2 that $Z(R)=(0)$; thus if $R$ is SP, $Q$ is regular and SP; hence by Proposition II.3, $Q$ is simple.

In view of the embedding theorem of $\S I$ II, every prime ring may be embedded in a simple ring.

Corollary 2. If $R$ is strongly prime, then Soc $R=(0)$ or $R$. (Recall that the socle (Soc) of a ring is the sum of its minimal right ideals.)

Proof. Suppose Soc $R \neq(0)$, but $R$ is SP. Then by [10, Proposition 7, p. 98], $Q$ is a full linear ring (a ring of endomorphisms of a vector space $V$ over a division ring $D$ ). Since $R$ is SP, $Q$ is simple-hence $\operatorname{Dim}_{D} V$ is fi- 
nite and $Q=M_{n}(D)$ for some $n$. Therefore $R$ is a right order in a matrix ring, and it follows from the version of the Faith-Utumi theorem given in [10] that any such ring with nonzero socle must be the matrix ring itself. So $R=M_{n}(D)$ and Soc $R=R$.

In $[6, p .14 a]$ it is suggested that every prime regular self-injective ring is a ring of quotients of a factor ring of a full linear ring. When one of these rings of quotients is simple, it is surely SP; it appears that it is not bounded SP. Set $R=D[x, y]$, the free algebra on two variables over the division ring $D$. Then $M_{n}(Q)=Q\left(M_{n}(R)\right)$ is bounded SP of bound 1 , and is not completely reducible as it contains elements $r$, $s$ such that $r s=1 \neq s r$.

Related to this question is the possible converse of IV.1. If $R$ is prime and $Q$ is simple (equivalently $Q$ is SP), is $R$ SP? Observe that if $Q$ is a matrix ring over a division ring, and $R$ is prime then $R$ is a right Goldie ring, and therefore SP.

V. Torsion theories. For the notions of torsion theories and related topics, see [11].

We adopt the notation $A \leq_{r} R$ to indicate that $A$ is a right ideal in $R$. An idempotent filter is a collection $\mathfrak{I}$ of right ideals in $R$ satisfying: 1. $R_{R} \in \mathfrak{D}$.

2. If $D \in \mathscr{D}, D \subset K$, and $K \leq_{r} R$, then $K \in \mathfrak{D}$.

3. If $D \in \mathcal{D}$, and $r \in R$, then $r^{-1} D \in \mathfrak{T}$.

4. If $D \in \mathfrak{D}, K \leq_{r} R$, and if for all $d \in D, d^{-1} K \in \mathscr{D}$, then $D \cap K \in \mathscr{D}$. From 3 and 4 we obtain

$4^{\prime}$. If $D, K \in \mathfrak{D}$, then $D \cap K \in \mathfrak{D}$.

A collection of right ideals satisfying $1,2,3$, and $4^{\prime}$ is called a topologizing filter. As is well known, there is a one-to-one correspondence between torsion theories and idempotent filters, and a similar correspondence between preidempotent filters and the left exact preradicals of [15].

We call an idempotent or topologizing filter $\mathfrak{D}$ proper if $(0) \notin \mathfrak{D}$.

Proposition V.l. In a strongly prime ring, every element of a proper idempotent or topologizing filter is an essential right ideal.

Proof. Let $D \in \mathscr{D}$, the topologizing filter. If there exists $r \in R^{\cdot}$ such that $r R \cap D=(0)$, then $r^{-1} 0=r^{-1} D \in \mathscr{I}$, by 3. If $\left\{s_{i}\right\}$ is an insulator for $r$, we have $\left(r s_{i}\right)^{-1} 0=s_{i}^{-1} r^{-1} 0$ for all $i$; hence by $4^{\prime}$,

$$
\bigcap_{i}\left(r s_{i}\right)^{-1} 0=\operatorname{Ann}\left(\left\{r s_{i}\right\}\right) \cdot \in \mathscr{D} \text {. }
$$

Thus $(0) \in \mathscr{D}$, so the filter is not proper. 
A right ideal $D$ is dense if, given $r \in R^{\circ}$ and $r^{\prime} \in R$, there exists $s \epsilon$ $R$ such that $r s \in R^{\circ}$ and $r^{\prime} s \in D$. A dense right ideal has zero as its left annihilator, and if $Z(R)=(0)$, then every essential right ideal is dense.

(0) and $R$ are the only torsion ideals of $R$ (in the sense of [11]) if and only if every proper idempotent filter consists of dense right ideals, and this occurs if and only if $R$ possesses a unique maximal proper torsion theory (the largest torsion theory in which $R$ is torsion-free). Since every dense right ideal is essential, we obtain

Corollary 1. If $R$ is strongly prime, then there exists a unique maximal proper torsion theory on $\operatorname{Mod}_{R}$.

Corollary 2. If $R$ is strongly prime, then (0) and $R$ are the only torsion ideals of $R$.

As pointed out by Rubin [14], the converse of Corollary 2 is not true; $Z / p^{2} Z$ has only two torsion ideals.

Let $M$ be a right $R$-module. Define an insulator for nonzero $m$ belonging to $M$ to be a finite subset of $R, S(m)$, such that zero is the right annihilator of $\{m s \mid s \in S(m)\} . M$ is a strongly prime module if every nonzero element of $M$ has an insulator. Since a nonzero SP module is necessarily faithful $(M r=$ (0) implies $r=0$ ), we see that $R$ is SP if and only if $R$ has a nonzero SP module. By proof similar to the ring case, we see that if $M$ is $S P$, then $Z(M)$ $=(0)$, and any essential extension of $M$ is SP.

Unfortunately, if $R$ is SP, the SP modules are exactly the nonsingular modules.

Corollary 3. If $R$ is strongly prime, then an $R$-module $M$ is strongly prime if and only if $Z(M)=(0)$.

Proof. If $M$ is $S P$, then $Z(M)=(0)$ has been noted above. The class $\mathcal{C}$ of SP modules is closed under isomorphic images, submodules, direct products, and injective hulls. Hence by Proposition 0.3 of [11], $\mathcal{C}$ is a class of torsionfree modules for some torsion theory and is proper since $R \in \mathcal{C}$. Let $\mathcal{C}^{\prime}$ denote the collection of nonsingular modules which are the torsion-free modules in the unique proper maximal torsion theory of $R$. Therefore $\mathcal{C}^{\prime} \subset \mathcal{C}$ since the torsion theory corresponding to $C$ is proper; and because $Z(M)=(0)$ for all modules $M$ that are $S P$, we have $\mathcal{C} \subset \mathcal{C}^{\prime}$; thus $\mathcal{C}=\mathcal{C}^{\prime}$.

Proposition V.2. If $Z(R)=(0)$, and every proper topologizing filter consists of essential right ideals, then $R$ is strongly prime.

Proof. If there exists $r \in R$, such that $r$ has no insulator in $R$, we de- 
fine $\mathcal{E}$ to be the collection of right ideals in $R$,

$$
\mathcal{E}=\{\operatorname{Ann}(\{r s \mid s, \epsilon I\}) \mid I \text { is a finite subset of } R\} \text {. }
$$

Then $\mathcal{E}$ is closed under finite intersections, $(0) \notin \mathcal{E}$, and if $A \in \mathcal{G}$, then $t^{-1} A \in E$, for all $t \in R$. Define

$$
\mathbb{M}=\left\{A \leq_{r} R \mid \text { there exists } B \in \mathcal{E} \text { such that } B \subseteq A\right\} \text {. }
$$

$M$ is a proper topologizing filter, but $r^{-1} 0 \in \mathbb{N}$, since $r \cdot r^{-1} 0=(0)$, and $Z(R)=(0)$, and $r^{-1} 0$ must be essential as it belongs to $\pi$, we have $r=0$. So every nonzero element has an insulator; thus $R$ is SP.

In [5] Goldman defines a kernel functor to be a functor $U: \operatorname{Mod}_{R} \rightarrow \operatorname{Mod}_{R}$ such that (i) $U(M) \subset M$ for all $M \in \operatorname{Mod}_{R}$, and (ii) $U$ is left exact (called a left exact preradical in [15]). Rubin [14] defines an ATF (absolutely torsion-free) ring to be a ring such that for all kernel functors $U, U(R)=(0)$ or $R$. That use of the word 'torsion' does not coincide with the use here. By an astonishing coincidence, ATF is the same as SP.

A right ideal $A$ of $R$ is weakly essential, if for any finite subset $I$ of $R, \bigcap_{s \in I} s^{-1} A \neq(0)$.

Proposition V.3 [14, Theorem 1.7]. $R$ is ATF if and only if every weakly essential right ideal is dense.

Proposition V.4. The following are equivalent for a ring $R$ :

(a) $R$ is strongly prime.

(b) $R$ is absolutely torsion-free.

(c) Every weakly essential right ideal of $R$ is dense.

(d) $Z(R)=(0)$ and every proper topologizing filter consists of essential right ideals.

Proof. (a) equivalent to (d) is a consequence of V.1 and V.2, so it suffices to show (a) is equivalent to (c).

Suppose (a) holds. Let $A$ be a weakly essential right ideal of $R$. Since $Z(R)=(0)$ (Proposition II.2), it suffices to show $A$ is essential. Let $r \in R^{\circ}$; and $\left\{s_{i}\right\}$ be an insulator for $r$. Since $A$ is weakly essential there exists nonzero $t$ in $R$ such that $\left\{r s_{i} t\right\}_{i} \subset A$; at least one of the $r s_{i} t$ is nonzero by the insulator property; thus $r R \cap A \neq(0)$. Suppose (a) does not hold. Then there exists $r \in R^{\circ}$ such that for any finite subset $I$ of $R$ we have Ann ( $\left.\left.r s \mid s \in I\right\}\right)$ $\neq(0)$. Then:

(i) $r^{-1} 0$ is weakly essential: if $I$ is a finite subset of $R$, then

$$
\bigcap_{s \in I} s^{-1} r^{-1} 0=\bigcap_{s \in I}(r s)^{-1} 0=\operatorname{Ann}(\{r s \mid s \in I\}) \neq(0) .
$$


(ii) $r^{-1} 0$ is not dense: $r \cdot r^{-1} 0=(0)$ but no dense right ideal has a nonzero left annihilator.

VI. Questions. 1. We can define a semi-SP ring in the obvious manner (subdirect product of strongly prime rings), and the strongly prime radical may be defined analogously to the prime radical. Is there an elementwise characterization of the strongly prime radical?

2. Can an SP ring contain a nil ideal? ( $\$$ III)

3. Characterize strongly prime group rings. Let $N(g)$ denote the smallest normal subgroup of $G$ containing $g$. Then in III.1(a), we showed that if $g-1$ has an insulator in $R G$, then $N(g)$ is not locally finite. It is not difficult to prove that if $g-1$ has no insulator in $R G$, then $N(g)$ is torsion. It may be that torsion should replace locally finite in the characterization of SP group rings.

4. Page [13] defines a prime module as one whose right annihilator (in $R$ ) is the same as that of any of its nonzero submodules. The faithful prime modules form a torsion-free class, analogous to the SP modules in Corollary 3 of V.1. What can be said about the resulting torsion theory? If $R$ is primitive, socle-free and SP then $R$ has a faithful prime module that is not SP.

5. If $R$ is prime and its maximal ring of quotients is simple, then is $R$ strongly prime? ( $(\mathrm{IV})$ Related to this is the characterization of those rings whose only torsion ideals (in the sense of $[11]$ ) are $(0)$ and $R$. Conjecture: this condition is equivalent to $R / Z(R)$ being SP. Observe that if this condition holds and $Z(R)=(0)$, then the maximal ring of quotients is simple. Unfortunately, the converse does not hold: the ring of upper triangular matrices over a field contains another torsion ideal, yet its maximal ring of quotients is simple.

Added in proof. 1. L. Small has answered question 2 in the affirmative.

2. Applications of this work to the classification of simple self-injective rings will appear in a joint paper by $\mathrm{K}$. Goodearl and the first author.

\section{REFERENCES}

1. I. G. Connell, On the group ring, Canad. J. Math. 15 (1963), 650-685. MR $27 \# 3666$.

2. C. C. Faith, Lectures on injective modules and quotient rings, Lecture Notes in Math., no. 49, Springer-Verlag, Berlin and New York, 1967. MR 37 \#2791.

3. E. Formanek, Group rings of free products are primitive, J. Algebra 26 (1973), 508-511.

4. A. W. Goldie, Torsion-free modules and rings, J. Algebra 1 (1964), 268-287. MR $29 \# 2282$. 
5. O. Goldman, Rings and modules of quotients, J. Algebra 13 (1969), 10-47. MR $39 \# 6914$.

6. K. R. Goodearl, Prime ideals in regular self-injective rings, Canad. J. Math. 25 (1973), 829-839.

7. I. N. Herstein, Noncommutative rings, Carus Math. Monographs, no. 15, Math. Assoc. of Amer; distributed by Wiley, New York, 1968. MR 37 \#2790.

8. N. Jacobson, Structure of rings, rev. ed., Amer. Math. Soc. Colloq. Publ., vol. 37, Amer. Math. Soc., Providence, R. I., 1964. MR 36 \#5158.

9. I. Kaplansky, Algebraic and analytic aspects of operator algebras, Conf. Board Math. Sci, Regional Conf. Ser. in Math., no. 1, Amer. Math. Soc., Providence, R. I., 1970. MR $47 \# 845$.

10. J. Lambek, Lectures on rings and modules, Blaisdell, Waltham, Mass., 1966. MR 34 \#5857.

11. - Torsion theories, additive semantics and rings of quotients, Lecture Notes in Math., vol. 177, Springer-Verlag, Berlin and New York, 1971. MR 44 $\# 1685$.

12. J. Lawrence, Primitivr group rings, Master's Thesis, McGill Univ., Montreal, Canada, 1973.

13. S. Page, Properties of quotient rings, Canad. J. Math. 24 (1972), 1122-1128. MR $47 \# 262$. 514.

14. R. A. Rubin, Absolutely torsion-free rings, Pacific J. Math. 46 (1973), 503-

15. B. T. Stenstrom, Rings and modules of quotients, Lecture Notes in Math., vol. 237, Springer-Verlag, Berlin and New York, 1971. MR 48 \#4010.

16. J. Viola-Prioli, On absolutely torsion-free rings and kernel functors, Ph.D. Thesis, Rutgers University, New Brunswick, N. J., 1973.

17. P. M. Cohn, On the free product of associative rings, Math. Z. 71 (1959), 380-398. MR $21 \# 5648$.

18. - On the free product of associative rings. II. The case of (skew) fields, Math. Z. 73 (1960), 433-456. MR 22 \#4747.

DEPARTMENT OF MATHEMATICS, MCGILL UNIVERSITY, MONTREAL, QUEBEC, CANADA

DEPARTMENT OF MATHEMATICS, CARLETON UNIVERSITY, OTTAWA, ONTARIO, CANADA

Current address (David Handelman): University of Giessen, Giessen, Federal Republic of Germany

Current address (John Lawrence): University of Chicago, Chicago, Illinois 60637 